\title{
Changes in Inflammation, Oxidative Stress, Mitochondrial DNA Content after Rosiglitazone in HIV Lipoatrophy
}

Marisa Tungsiripat ${ }^{1}$, Dalia El-Bejjani ${ }^{1}$, Nesrine Rizk ${ }^{2}$, Bo Hu${ }^{1}$, Allison C Ross ${ }^{2}$, Ulrich A Walker ${ }^{3,4}$, Dirk Lebrecht ${ }^{3}$, Ginger Milne ${ }^{5}$, Norma Storer ${ }^{2}$ and Grace A McComsey ${ }^{*}$

${ }^{1}$ Cleveland Clinic, Cleveland, $\mathrm{OH}, \mathrm{USA}$

${ }^{2}$ Case Western Reserve University, Cleveland, $\mathrm{OH}$, USA

${ }^{3}$ Albert-Ludwigs University, Freiburg, Germany

${ }^{4}$ Department of Rheumatology, Basel, Switzerland

${ }^{5}$ Vanderbilt University School of Medicine, Nashville, TN, USA

\begin{abstract}
Objective: We aim to evaluate the mechanisms of rosiglitazone-induced fat recovery in HIV+ patients with lipoatrophy on thymidine Nucleoside Reverse Transcriptase Inhibitors (NRTI) sparing regimens.

Method: Measures of limb fat (DXA), oxidative stress (F2 isoprostanes) and inflammation [High-sensitivity $\mathrm{C}$ - reactive protein (hsCRP), soluble Tumor Necrosis Factor Receptors (sTNFR)-I, sTNFR-II, and interleukin (IL)-6] were performed. Gluteal fat mitochondrial DNA (mtDNA) and peroxisome proliferator-activated receptor (PPAR)- $\mathrm{Y}$ RNA [expressed as PPAR-y/Glyceraldehyde 6-Phosphate Dehydrogenase (GAPDH) RNA ratio] were measured by quantitative PCR.

Result: 71 patients on thymidine NRTI-sparing regimens were randomized to rosiglitazone vs. placebo for 48 weeks. Duration off thymidine NRTIs was similar between groups. From week $0-48$, limb fat increased significantly $(p=0.02)$ more in the rosiglitazone than in the placebo group. Within both groups, F2-isoprostanes, sTNFR-I and sTNFR-II increased significantly ( $p \leq 0.003)$, hsCRP decreased significantly $(\leq 0.02)$, and IL-6 did not change. No differences were seen between groups in any of the inflammation markers. Fat mtDNA (copies/ cell) increased nonsignificantly: $+41(p=0.08)$ and $+29(p=0.38)$ within rosiglitazone and placebo group; respectively. PPAR- $\gamma /$ GAPDH ratio did not change within or between groups.

Conclusion: Limb fat improvements seen after rosiglitazone were not associated with changes in mtDNA, oxidative or inflammation markers, or PPAR-y expression. F2 isoprostanes and some of the inflammation markers worsened over time in these subjects on stable ART, regardless of the rosiglitazone assignment. Thus, lipoatrophy can be in part overcome by a separate pathway independent of mitochondrial DNA depletion, such as PPAR-y.
\end{abstract}

Keywords: AntiRetroviral therapy; Fat loss; Lipoatrophy; Mitochondrial DNA; Oxidative stress; Rosiglitazone

\section{Introduction}

Lipoatrophy is subcutaneous fat wasting of the face, arms, buttocks and/or legs which has been described in HIV-infected individuals with or without associated central fat accumulation, insulin resistance, and dyslipidemia. While sufficiently distressing from a cosmetic standpoint alone, lipoatrophy can be stigmatizing as a prominent and visible association with HIV/ AIDS. Furthermore, lipoatrophy decreases the quality of life of HIV-infected individuals and threatens the effectiveness of AntiRetroviral Therapy (ART) [1].

The mechanism of antiretroviral-associated lipoatrophy remains poorly defined. Data has increasingly implicated thymidine Nucleoside Reverse Transcriptase Inhibitors (NRTI) via mitochondrial toxicity [24]. As NRTI down-regulation of the peroxisome proliferator-activated receptor (PPAR) $-\gamma$ has also been implicated as a cause in vitro [4], thiazolidenediones, potent agonists of PPAR- $\gamma$, would be expected to reverse lipoatrophy. However, these data have been conflicting; but, most of these studies had not excluded thymidine NRTI's [5-11]. Concurrent usage of rosiglitazone and thymidine NRTIs has been shown to blunt rosiglitazone's activity on PPAR- $\gamma$ [12]. We previously reported that rosiglitazone improved lipoatrophy in HIV-infected patients with lipoatrophy in the setting of thymidine NRTI-sparing regimens [13]. In this present substudy, we have aimed to evaluate the underlying mechanisms of these fat changes with rosiglitazone.
Gaining understanding of the underlying causes of lipoatrophy would help target new treatment options. In addition to the increasingly clear implication of thymidine NRTIs [2-4], measures of oxidative stress and inflammation have also been associated with lipoatrophy. Elevated F2 isoprostanes levels have been associated with lipoatrophy [14]. The prostaglandin-like compounds F2 isoprostanes are accurate measures of oxidative stress in vivo [15]. Lipoatrophy has also been associated with abnormal inflammatory markers in comparison to individuals without lipoatrophy [16]. In order to gain insight into the underlying mechanisms of the observed fat changes after rosiglitazone in these subjects with ART-associated lipoatrophy, we evaluated changes in oxidative and inflammation markers, mitochondrial $(\mathrm{mt})$ DNA levels, and PPAR- $\gamma$ transcripts.

*Corresponding author: Grace A McComsey, Professor of Pediatrics and Medicine Case School of Medicine, Cleveland, OH 44106, USA, Tel: 216844 3607; Fax: 216844 8362; E-mail: Grace.mccomsey@case.edu

Received August 30, 2012; Accepted October 10, 2012; Published October 15 2012

Citation: Tungsiripat M, El-Bejiani D, Rizk N, Hu B, Ross AC, et al. (2012) Changes in Inflammation, Oxidative Stress, Mitochondrial DNA Content after Rosiglitazone in HIV Lipoatrophy. J AIDS Clinic Res 3:174. doi:10.4172/2155-6113.1000174

Copyright: (C) 2012 Tungsiripat M, et al. This is an open-access article distributed under the terms of the Creative Commons Attribution License, which permits unrestricted use, distribution, and reproduction in any medium, provided the original author and source are credited. 


\section{Materials and Methods}

\section{Subjects}

This randomized double-blind placebo-controlled trial evaluated baseline and 48-week measurements of limb fat (DXA), oxidative stress (F2 isoprostanes), inflammation [high-sensitivity C-Reactive Protein (hsCRP), soluble Tumor Necrosis Factor Receptors (sTNFR-)I, sTNFRII, and InterLeukin (IL)-6], and gluteal fat mitochondrial indices [DNA and PPAR- $\gamma$ RNA expressed as PPAR- $\gamma /$ GlycerAldehyde 6-Phosphate DeHydrogenase (GAPDH) RNA ratio] in HIV-infected patients with lipoatrophy receiving rosiglitazone or placebo for 48 weeks. The participants were enrolled at the John T. Carey Special Immunology Unit of University Hospitals Case Medical Center and at the Cleveland Clinic in Cleveland, Ohio, USA. The Institutional Review Board (IRB) Committees of both institutions approved the study. All patients gave written informed consent.

\section{Study Population}

HIV-infected men and women $\geq 18$ years old with clinical lipoatrophy were enrolled. Clinical lipoatrophy was defined as fat loss of at least moderate severity in at least two different areas of the following body areas: face, arms, legs, or buttocks. Self-reports were confirmed by a physician. Inclusion criteria included a past history of receiving thymidine NRTI (stavudine or zidovudine) for at least 12 cumulative months, discontinuation of thymidine NRTI therapy and receipt of a stable thymidine NRTI-sparing regimen for at least 24 weeks prior to study entry, HIV-1 RNA $\leq 5000$ copies $/ \mathrm{mL}$, and no intent on the part of the subject or provider to alter ART over the study period. In addition, women of childbearing potential were required to have a negative pregnancy test at study entry and to use strict contraception during the study.

Individuals were excluded if they had liver cirrhosis, heart failure of New York Heart Association class 3 or 4, diabetes mellitus, or were receiving metformin or glitazones. Individuals who were pregnant or breastfeeding, or who were receiving any hormonal supplementation with recombinant growth hormone, anabolic steroids, estrogen or testosterone (except at replacement doses) were excluded. Additionally, subjects were excluded if they had serum transaminases greater than 2 times the Upper Limit of Normal (ULN), lipase > 2.5 ULN, creatinine $>3$ ULN, PT/PTT greater than 1.2 ULN, absolute neutrophil $<750 /$ $\mathrm{mm}^{3}$, hemoglobin $<9.0 \mathrm{~g} / \mathrm{dL}$, platelet count $<75,000 / \mathrm{mm}^{3}$, or glucose $<70 \mathrm{mg} / \mathrm{dL}$.

\section{Intervention}

The subjects were centrally randomized in a double-blinded fashion to receive either rosiglitazone or matching placebo for 48 weeks. Both the study drug and matching placebo were provided by GlaxoSmithKline, Research Triangle, NC. In the dose-escalation period, subjects received rosiglitazone $4 \mathrm{mg}$ daily for 4 weeks. The dose was then increased to $4 \mathrm{mg}$ twice daily for the remainder of the study. All subjects tolerated the lead-in period and none dropped out during the lead-in period. Participants continued their present antiretroviral regimens and were advised to maintain their current diet and exercise habits.

All visits included assessment for clinical adverse events, use of concomitant medications, targeted physical examination and complete blood count, biochemistry (including electrolytes, liver transaminase levels, and creatinine concentration). In addition, CD4+ cell count and HIV-1 RNA were concomitantly measured as markers of HIV disease status. Adherence to study medication was determined by pill count of dispensed versus returned pills at each study visit. Permanent cessation of the drug was mandatory for grade 4 adverse events or pregnancy.

\section{Assessment of changes in body fat}

Assessments of changes in body fat included physical examination and whole body Dual-energy X-ray Absorptiometry (DXA) at study entry, weeks 24 , and week 48 . To evaluate subjective assessments of fat changes by both physicians and subjects, we evaluated the changes in clinical lipoatrophy scores. Clinical lipoatrophy scores were obtained by questionnaires at study entry and at weeks 24 and 48 . These questionnaires rated the loss of fat in predefined body areas: arms, legs, buttocks and the face. Assessments within each of these sites were rated " 0 " for "absent", " 1 " for "mild", " 2 " for "moderate", and " 3 " for "severe". These scores were summed for a lipoatrophy self-rated score. Thus, the lipoatrophy score could vary between $0-12$. The same questionnaire was completed separately and independently by the subject and the physician.

Total body DXA scans were performed at a single site (Case) on all study subjects using a Hologic QDR-4500A (Hologic Inc, Bedford, MA). The DXA were assessed with a dedicated scanner and technologist who were blinded to treatment allocation. Analysis of overall and body site-specific fat were performed on the basis of the standard protocol for body composition examination.

\section{Oxidative stress}

Measures of oxidative stress (F2 isoprostanes) were assessed at study entry and week 48 . Blood was drawn into tubes containing EDTA and immediately centrifuged to separate the plasma. An aliquot of plasma was then removed and immediately stored at $-70^{\circ} \mathrm{C}$ until measurements of F2-isoprostanes were performed at the Vanderbilt University laboratory. The $\mathrm{F}_{2}$-isoprostanes are quantified in biologic fluids after Sep-Pak and TLC purification as pentafluorobenzyl ester, trimethylsilyl ether derivatives utilizing stable isotope dilution techniques with deuterated 15-F2t-IsoP (Cayman Chemical, Ann Arbor, MI) as an internal standard. The precision of the assay is $\pm 4 \%$, the accuracy $\pm 95 \%$, and interassay variability is less than $8 \%$ [14].

\section{Inflammatory markers}

At weeks 0 and 48, the following biomarkers were measured in blood: hsCRP, sTNFR-I and -II, and IL-6. The markers were measured in duplicate and averaged using commercially available enzymelabeled immunosorbent sandwich assays (Searchlight; Thermo Fisher Scientific, Wobern, MA). The median intra-assay coefficients of variation for hsCRP, sTNFR-I, sTNFR-II, and IL-6 were 6.9\%, 8.8\%, $8.6 \%$, and $11.7 \%$ respectively. The median interassay coefficients of variation for each assay were $4.5 \%, 10.8 \%, 6.0 \%$, and $10.4 \%$, respectively.

\section{Tissue sampling and measures of fat mtDNA and PPAR- $\gamma$ RNA}

Study subjects underwent gluteal fat biopsies at study entry and week 48. Fat biopsies were optional and not mandatory for enrollment into the study. The fat was obtained by $6-\mathrm{mm}$ skin punch biopsies under local anesthesia and trimmed from under the skin portion. All tissue samples were stored in a $-70^{\circ} \mathrm{C}$ freezer at the local site. Fat mtDNA and PPAR $-\gamma$ RNA (expressed as PPAR- $\gamma /$ GAPDH RNA ratio) were evaluated by real-time quantitative PCR. The mtDNA assays were performed on batched specimens at the end of the study as described previously [17]. The laboratory personnel were blinded to all sample characteristics. Briefly, total DNA was extracted with the 
QIAamp DNA isolation kit (Quiagen, Hilden, Germany). MtDNA and nDNA copy numbers were determined by quantitative PCR using the ABI 7700 sequence detection system (Applied Biosystems, Foster City, CA) .The mtDNA ATP- 6 gene was amplified and quantified with a FAM-fluorophore labeled probe. For the quantification of nDNA, exon 8 of the GAPDH-gene was amplified and quantified using a VICfluorophore labeled probe. Amplifications of mitochondrial and nuclear products were performed separately in optical 96-well plates (Applied Biosystems). All samples were run in triplicate. Absolute mtDNA and nDNA copy numbers were calculated using serial dilutions of plasmids with known copy numbers of mtDNA and nDNA.

RNA was extracted (RNeasy Kit, Qiagen, Hilden, Germany) and the quantity and integrity of the RNA were verified using RNA 6000 nano chips (Agilent 2100 Bioanalyser, Palo Alto, CA). $5 \mu \mathrm{g}$ RNA from each sample was reverse transcribed using 200 U Superscript II (Invitrogen, Carlsbad, CA) and $100 \mu \mathrm{M}$ oligo (dT) (Invitrogen Carlsbad, CA) as primer.

Gene expression was quantified using the Light Cycler 480 (Roche, Germany) on a 384 well plate. $10 \mu \mathrm{l}$ reactions contained $5 \mu \mathrm{l}$ of SYBR Green I Master mix (Roche, Mannheim, Germany), 50 ng cDNA template and $0.5 \mu \mathrm{M}$ of each primer. Target gene PPAR- $\gamma$ was amplified (forward 5'-GACCTGAAACTTCAAGAGTACCAAA; reverse $5^{\prime}$-TGAGGCTTATTGTAGAGCTGAGTC) in duplicate and the light cycling conditions were as follows: activation $\left(95^{\circ} \mathrm{C}\right.$ for $10 \mathrm{sec}$.), 40 amplification cycles $\left(95^{\circ} \mathrm{C}\right.$ for 10 seconds, $52^{\circ} \mathrm{C}$ for 5 seconds, and $72^{\circ} \mathrm{C}$ for 12 seconds). Melting curve analysis was done to ensure that the investigatedgeneswererepresentedbyasinglepeak, indicatingspecificity. Gene expression was calculated from the real-time PCR efficiency in relation to GAPDH gene (forward 5' - TGCACCACCAACTGCTTAG; reverse 5'-AGAAACCGACCTGGATTGCTC).

\section{Statistical analysis}

Continuous variables were summarized as means and standard deviations or median and inter-quartile ranges, and categorical variables were summarized as frequencies and percentages. T-tests and Wilcoxon tests were used to compare the change of variables over time within and between groups as appropriate. Pearson correlation was calculated to assess the association between variables. All p-values are two-sided. All analyses were carried out by using SAS 9.2 (Cary, NC).

\section{Results}

Seventy-one patients with clinical lipoatrophy were enrolled; 57 agreed to enroll in the biopsy substudy. Baseline characteristics were similar in those who enrolled in the biopsy study versus those who refused to have the biopsies performed. Seventeen percent were female; $51 \%$ were white. The baseline parameters were similar between the two groups and detailed in Table 1. The durations off thymidine NRTIs were similar between the rosiglitazone and placebo groups: 47 vs. 42 months, respectively.

At 48 weeks, DXA-measured limb fat increased significantly ( $\mathrm{p}=0.02$ ) more in the rosiglitazone than in the placebo group: 448 grams vs. 153 grams, respectively as detailed in Table 2 . The increases within both groups from baseline to 48 weeks were also significant $(\mathrm{p}<0.03)$. Facial atrophy was evaluated clinically. The clinical lipoatrophy scores have been reported previously [13]. There was no difference in facial atrophy scores between the groups in either subject or physician scores at 24 or 48 weeks. Within only the rosiglitazone group, subject facial lipoatrophy scores changed significantly $(\mathrm{p}<0.001)$, from a baseline score median (IQR) $2(1,2)$ by $-1(-1,0)$ at 48 weeks.
The median F2 isoprostanes, sTNFR-I and sTNFR-II levels increased significantly $(\mathrm{p} \leq 0.003)$ within both groups over the 48 weeks of the study but not between the groups. hsCRP decreased significantly $(\leq 0.02)$ within both but not between the groups. IL-6 levels did not change within or between groups. In the 57 patients with fat biopsy results, the fat mtDNA content were assessed. The median values were not significantly $(\mathrm{p}=0.58)$ different at study entry: 168 copies/ cell in the rosiglitazone group vs.205 copies/cell in the placebo group. Over 48 weeks, the median fat mtDNA values trended upwards approaching significance in the rosiglitazone group: $+41(\mathrm{p}=0.08)$ in the rosiglitazone group vs. +29 ( $\mathrm{p}=0.38)$ in the placebo group, respectively. The changes in mtDNA did not correlate with change in hsCRP.

The PPAR- $\gamma /$ GAPDH ratio decreased by a non significant $6.8 \%$ and $0.5 \%$ within the rosiglitazone and placebo groups, respectively. Percent changes in PPAR- $\gamma /$ GAPDH ratio did not significantly correlate with either changes in limb fat $(r=-0.17, p=0.33)$ or mtDNA $(r=-0.01, p$ $=0.98)$. Changes in F2-isoprostanes correlated significantly $(\mathrm{r}=0.35, \mathrm{p}$ $=0.011)$ with changes in limb fat. The correlation between changes in hsCRP and changes in limb fat approached significance $(r=0.27, p=$ 0.052 ), reflecting a smaller decrease in hsCRP correlating with a larger increase in fat limb. Changes in other inflammation markers did not correlate with changes in limb fat. None of the subjects reported adverse events as a result of the procedures. HIV-1 RNA levels remained stable throughout the study. At week $48,98 \%$ of the study subjects had HIV-1 RNA levels less than 400 copies/ mL.

\section{Discussion}

We have evaluated rosiglitazone's effect on fat changes in HIVinfected patients with lipoatrophy who were receiving thymidine NRTI sparing regimens. We previously reported that lipoatrophy improved after rosiglitazone at 48 weeks in comparison to placebo in the primary study [13]. Our finding of improvement of lipoatrophy from baseline

\begin{tabular}{|c|c|c|}
\hline Median (IQR) & Rosiglitazone $(n=34)$ & Placebo $(n=37)$ \\
\hline Age in years & $47(44,52)$ & $52(44,54)$ \\
\hline Men, N (\%) & $29(85)$ & $30(81)$ \\
\hline White, N (\%) & $19(56)$ & $17(45)$ \\
\hline CD4+ cell count, cells $/ \mathrm{mm}^{3}$ & $596(437,706)$ & $690(422,860)$ \\
\hline Nadir CD4+ cell count, cells $/ \mathrm{mm}^{3}$ & $205(98,271)$ & $123(64,247)$ \\
\hline HIV-1 RNA <50 copies/mL, N (\%) & $28(82)$ & $29(78)$ \\
\hline Duration HIV diagnosis (months) & $146(122,194)$ & $170(122,231)$ \\
\hline Duration on ART (months) & $114(96,127)$ & $105(74,123)$ \\
\hline Duration on tNRTI (months) & $65(48,59)$ & $69(24,83)$ \\
\hline Duration off tNRTI (months) & $47(14,59)$ & $42(11,64)$ \\
\hline NNRTI at study entry, N (\%) & $15(44)$ & $14(37)$ \\
\hline $\mathrm{PI}$ at study entry, $\mathrm{N}(\%)$ & $19(56)$ & $23(62)$ \\
\hline Limb fat, grams & $4695.9(3643.6,7758.3)$ & $\begin{array}{l}5966.7 \text { (3514.3, } \\
8434.7)\end{array}$ \\
\hline $\mathrm{BMI}, \mathrm{kg} / \mathrm{m}^{2}$ & $25.3(22.5,30.8)$ & $25.8(22.8,27.9)$ \\
\hline sTNFR-I, pg/mL & $773.5(597.0,957.0)$ & $\begin{array}{l}701.5 \text { (573.1, } \\
891.0)\end{array}$ \\
\hline sTNFR-II, pg/mL & $564.0(232.0,1247.0)$ & $\begin{array}{l}605.0(315.0 \\
1208.2)\end{array}$ \\
\hline IL-6, pg/mL & $6.0(2.0,13.3)$ & $6.0(3.0,10.5)$ \\
\hline hs CRP, mg/L & $7.6(4.1,18.1)$ & $3.2(1.2,14.7)$ \\
\hline $\mathrm{F} 2$ isoprostanes, $\mathrm{ng} / \mathrm{mL}$ & $0.032(0.024,0.043)$ & $\begin{array}{l}0.032(0.027 \\
0.046)\end{array}$ \\
\hline mtDNA, copies/ cell & $168(143,214)$ & $205(166,222)$ \\
\hline
\end{tabular}

\# Significant difference between the groups $(p=0.04)$

Table 1: Baseline characteristics. 


\begin{tabular}{|c|c|c|c|c|c|}
\hline Median (IQR) & Rosiglitazone $(n=29)$ & $\mathrm{P}$ (within group) & Placebo $(n=32)$ & $\mathrm{P}$ (within group) & $\mathrm{P}$ (between groups) \\
\hline Limb fat, grams & $+448(138,1670)$ & $<0.001$ & $+153(-100,682)$ & $<0.03$ & 0.02 \\
\hline $\mathrm{BMI}, \mathrm{kg} / \mathrm{m}^{2}$ & $+0.9(0.08,1.6)$ & $<0.001$ & $+0.5(-0.08,1.3)$ & 0.03 & 0.24 \\
\hline $\mathrm{F} 2$ isoprostanes, $\mathrm{ng} / \mathrm{mL}$ & $+0.019(-0.002,0.036)$ & 0.003 & $+0.017(-0.003,0.032)$ & 0.001 & 0.078 \\
\hline sTNFR-I, pg/mL & +733.8 (318.5, 9891.3) & $<0.001$ & +973.3 (373.3, 8662.9) & $<0.001$ & 0.98 \\
\hline sTNFR-II, pg/mL & +1722.4 (872.9, 5951.9) & $<0.001$ & +1586.9 $(994.7,5552.8)$ & $<0.001$ & 0.97 \\
\hline $\mathrm{IL}-6, \mathrm{pg} / \mathrm{mL}$ & $+0.2(-9.0,34.2)$ & 0.30 & $+1.4(-5.2,16.9)$ & 0.29 & 0.92 \\
\hline hs CRP, mg/L & $-4.6(-11.1,-0.9)$ & $<0.001$ & $-1.5(-8.8,0.18)$ & 0.02 & 0.11 \\
\hline mtDNA, copies/cell $(n=57)$ & $+41(-14,66)(n=27)$ & 0.08 & $+29(-36,67)(n=30)$ & 0.38 & 0.54 \\
\hline PPAR- $\gamma /$ GAPDH ratio $(n=57)$ & $-6.8 \%(-7.2 \%,-0.4 \%)(n=27)$ & 0.32 & $-0.5 \%(-7.4 \%, 4.3 \%)(n=30)$ & 0.86 & 0.54 \\
\hline
\end{tabular}

Table 2: Changes over 48 weeks.

in both arms is consistent with discontinuation of tNRTI as reported in prior studies [2,3,18-20]. In addition, lipoatrophy improvements were significantly greater in the rosiglitazone arm, consistent with an effect of the study drug beyond that of tNTRI discontinuation. Additionally, variations in inflammatory markers were observed as previously reported [21]. Several of the inflammatory markers (hsCRP, sTNFRI, sTNFRII) did not differ significantly between arms, but changed significantly in both arms over time and independent of lipoatrophy status as expected and consistent with prior studies [22-24]. In this present substudy, we evaluated oxidative and inflammation and mitochondrial indices in these subjects in order to evaluate the underlying mechanisms of these observed fat changes. Fat mtDNA and mtDNA-encoded protein levels have been shown to be decreased in subcutaneous fat tissue from HIV-infected individuals with lipoatrophy receiving thymidine NRTI [25-27]. MtDNA levels increased after discontinuation of tNTRI [26]. However, in our study during which DXA-measured limb fat increased after rosiglitazone for 48 weeks in patients receiving thymidine NRTI-sparing regimens, we did not detect significant differences in fat mtDNA levels or PPAR- $\gamma$ transcripts. Similarly, though possibly related to concomitant thymidine NRTI exposure, Mallon and others also observed no difference between rosiglitazone and placebo in adipocyte mtDNA or PPAR- $\gamma$ gene expression [12].

Although the fat biopsy investigations which were assessed in this study did not clearly elucidate the mechanism of the concurrently observed changes in DXA-measured limb fat, mtDNA levels did trend upwards from baseline to 48 weeks. This increase did approach significance $(p=0.08)$ in the rosiglitazone group. Thus, reversal of mitochondrial content may have been a factor in the observed improvement of lipoatrophy in these subjects. This observation is consistent with prior studies which showed that mitochondrial DNA and RNA alterations were linked to antiretroviral-associated lipoatrophy $[2,28]$.

F2 isoprostanes are formed from peroxidation of essential fatty acids and are an accurate measure of oxidative stress in vivo [15]. Levels of F2 isoprostanes increased over the 48 weeks of the study regardless of the rosiglitazone assignment. These increases significantly correlated with the observed changes in limb fat. As elevated F2- isoprostanes have been associated with lipoatrophy in comparison to individuals without lipoatrophy [14], we would have expected F2 isoprostanes levels to improve or decrease in the setting of improvement in lipoatrophy. However, the levels increased over time in these study subjects. F2 isoprostane levels have been reported to increase with age in HIV-negative individuals [29]. While aging may be a possible mechanism to explain this increase, it does not likely explain fully this observed increase in F2 isoprostane level over the 48 week study given the relatively short duration of the study. In HIV-infected individuals, F2 isoprostanes may be associated with viremia. F2 isoprostanes have been reported to be higher in HIV-infected subjects with lower viral loads [30] and to increase over time in subjects initiating ART [31]. Additional studies would be needed to characterize oxidant stress in HIV-infected individuals. To our knowledge, oxidative markers have not been previously investigated or reported in HIV-infected subjects on stable ART.

Some of the inflammation markers also worsened over time in these subjects on stable ART. This finding is consistent with the results of prior studies [22-24]. However, two of these prior studies suggest that the increased immune activation and enhanced inflammatory state are related to uncontrolled viremia. In contrast in our trial, the TNF-receptors increased significantly over 48 weeks in the setting of continuous ART and controlled viremia. We acknowledge that there are limitations to this study. The investigations of mitochondrial indices, oxidative and inflammatory markers presented here were secondary endpoints of a trial designed and powered to evaluate changes in DXA-measured limb fat. Although our data do not support that the observed changes in limb fat were associated with changes in mitochondrial indices, we cannot completely preclude a smaller effect. Although relatively small sample size is a limitation, the biopsies and markers were obtained in a randomized trial over 48 weeks with consistent outcomes.

\section{Conclusion}

In summary, in our study of HIV-infected patients with lipoatrophy, limb fat improvement was seen after rosiglitazone. However, mitochondrial indices, oxidative, and inflammatory markers were no different between those who received rosiglitazone and those who did not. This suggest that lipoatrophy may in part be able to be overcome by a separate pathway independent of mitochondrial DNA depletion, such as the PPAR- $\gamma$ pathway. Further studies are needed to further define the mechanism of ART-associated lipoatrophy.

\section{Acknowledgements}

The study was supported in part by NIAID AI-060484 (GM) and Al-070078 (MT), GlaxoSmithKline Collaborative Studies, the NCRR CTSA 1UL1RR024989 (Cleveland, $\mathrm{OH}$ ) and the clinical Core of the Case Center for AIDS Research Al36219.

\section{Conflict of Interest}

MT- Research grant from Bristol-Myers Squibb. AC-Research grant from Bristol-Myers Squibb, GlaxoSmithKline and Cubist Pharmaceuticals; GAMConsultant, speaker, research grants from GlaxoSmithKline, Bristol-Myers Squibb Gilead Sciences, and Abbott Labs. DEB, NR, VD, MAO, NS, DN- no conflicts.

We especially thank our patients who participated in this study.

\section{References}

1. Dukers NH, Stolte IG, Albrecht N, Coutinho RA, de Wit JB (2001) The impact of experiencing lipodystrophy on the sexual behaviour and well-being among HIV-infected homosexual men. Aids 15: 812-813.

2. McComsey GA, Paulsen DM, Lonergan JT, Hessenthaler SM, Hoppel CL, 
Citation: Tungsiripat M, El-Bejjani D, Rizk N, Hu B, Ross AC, et al. (2012) Changes in Inflammation, Oxidative Stress, Mitochondrial DNA Content after Rosiglitazone in HIV Lipoatrophy. J AIDS Clinic Res 3:174. doi:10.4172/2155-6113.1000174

et al. (2005) Improvements in lipoatrophy, mitochondrial DNA levels and fat apoptosis after replacing stavudine with abacavir or zidovudine. Aids 19: 15-23.

3. Milinkovic A, Martinez E, López S, de Lazzari E, Miró O, et al. (2007) The impact of reducing stavudine dose versus switching to tenofovir on plasma lipids, body composition and mitochondrial function in HIV-infected patients. Antivir Ther 12: 407-415

4. Viengchareun S, Caron M, Auclair M, Kim MJ, Frachon P, et al. (2007) Mitochondrial toxicity of indinavir, stavudine and zidovudine involves multiple cellular targets in white and brown adipocytes. Antivir Ther 12: 919-929.

5. Carr A, Workman C, Carey D, Rogers G, Martin A, et al. (2004) No effect of rosiglitazone for treatment of HIV-1 lipoatrophy: randomised, double-blind, placebo-controlled trial. Lancet 363: 429-438.

6. Cavalcanti RB, Raboud J, Shen S, Kain KC, Cheung A, et al. (2007) A randomized, placebo-controlled trial of rosiglitazone for HIV-related lipoatrophy. $\mathrm{J}$ Infect Dis 195: 1754-1761.

7. Hadigan C, Yawetz S, Thomas A, Havers F, Sax PE, et al. (2004) Metabolic effects of rosiglitazone in HIV lipodystrophy: a randomized, controlled trial. Ann Intern Med 140: 786-794.

8. Mulligan K, Yang Y, Wininger DA, Koletar SL, Parker RA, et al. (2007) Effects of metformin and rosiglitazone in HIV-infected patients with hyperinsulinemia and elevated waist/hip ratio. Aids 21: 47-57.

9. Slama L, Lanoy E, Valantin MA, Bastard JP, Chermak A, et al. (2008) Effect of pioglitazone on HIV-1-related lipodystrophy: a randomized double-blind placebo-controlled trial (ANRS 113). Antivir Ther 13: 67-76.

10. Sutinen J, Häkkinen AM, Westerbacka J, Seppälä-Lindroos A, Vehkavaara S et al. (2003) Rosiglitazone in the treatment of HAART-associated lipodystrophy-a randomized double-blind placebo-controlled study. Antivir Ther 8: 199-207.

11. van Wijk JP, de Koning EJ, Cabezas MC, op't Roodt J, Joven J, et al. (2005) Comparison of rosiglitazone and metformin for treating HIV lipodystrophy: a randomized trial. Ann Intern Med 143: 337-346.

12. Mallon PW, Sedwell R, Rogers G, Nolan D, Unemori P, et al. (2008) Effect of rosiglitazone on peroxisome proliferator-activated receptor gamma gene expression in human adipose tissue is limited by antiretroviral drug-induced mitochondrial dysfunction. J Infect Dis 198: 1794-1803.

13. Tungsiripat M, Bejjani DE, Rizk N, O'riordan MA, Ross AC, et al. (2010) Rosiglitazone improves lipoatrophy in patients receiving thymidine-sparing regimens. AIDS 24: 1291-1218.

14. McComsey GA, Morrow JD (2003) Lipid oxidative markers are significantly increased in lipoatrophy but not in sustained asymptomatic hyperlactatemia. J Acquir Immune Defic Syndr 34: 45-49.

15. Morrow JD, Roberts LJ (1996) The isoprostanes. Current knowledge and directions for future research. Biochem Pharmacol 51: 1-9.

16. Samaras K, Gan SK, Peake PW, Carr A, Campbell LV (2009) Proinflammatory markers, insulin sensitivity, and cardiometabolic risk factors in treated HIV infection. Obesity (Silver Spring) 17: 53-59.

17. Setzer B, Schlesier M, Walker UA (2005) Effects of of didanosine-related depletion of mtDNA in human T lymphocytes. J Infect Dis 191: 848-855.
18. Carr A, Workman C, Smith DE, Hoy J, Hudson J, et al. (2002) Abacavir substitution for nucleoside analogs in patients with HIV lipoatrophy: a randomized trial. Jama 288: 207-215.

19. Martin A, Smith DE, Carr A, Ringland C, Amin J, et al. (2004) Reversibility of lipoatrophy in HIV-infected patients 2 years after switching from a thymidine analogue to abacavir: the MITOX Extension Study. Aids 18: 1029-1036.

20. Moyle GJ, Sabin CA, Cartledge J, Johnson M, Wilkins E, et al. (2006) A randomized comparative trial of tenofovir DF or abacavir as replacement for a thymidine analogue in persons with lipoatrophy. Aids 20: 2043-2050.

21. Tungsiripat M, El-Bejjani D, Rizk N, Dogra V, O'Riordan MA, et al. (2011) Carotid Intima Media Thickness, Inflammatory Markers, and Endothelia Activation Markers in HIV Patients with Lipoatrophy Increased at 48 Weeks Regardless of Use of Rosiglitazone or Placebo. AIDS Res Hum Retroviruses 27: 295-302

22. Ross AC, Armentrout R, O'Riordan MA, Storer N, Rizk N, et al. (2008) Endothelial activation markers are linked to HIV status and are independent of antiretroviral therapy and lipoatrophy. J Acquir Immune Defic Syndr 49: 499506.

23. El-Sadr WM, Grund B, Neuhaus J, Babiker A, Cohen CJ, et al. (2008) Risk for opportunistic disease and death after reinitiating continuous antiretrovira therapy in patients with HIV previously receiving episodic therapy: a randomized trial. Ann Intern Med 149: 289-299.

24. Torriani FJ, Komarow L, Parker RA, Cotter BR, Currier JS, et al. (2008) Endothelial function in human immunodeficiency virus-infected antiretroviralnaive subjects before and after starting potent antiretroviral therapy: The ACTG (AIDS Clinical Trials Group) Study 5152s. J Am Coll Cardiol 52: 569-576.

25. Cherry CL, Gahan ME, McArthur JC, Lewin SR, Hoy JF, et al. (2002) Exposure to dideoxynucleosides is reflected in lowered mitochondrial DNA in subcutaneous fat. J Acquir Immune Defic Syndr 30: 271-277.

26. McComsey G, Southwell H, Gripshover B, Salata R, Valdez H (2003) Effect of antioxidants on glucose metabolism and plasma lipids in HIV-infected subjects with lipoatrophy. J Acquir Immune Defic Syndr 33: 605-607.

27. Nolan D, Mallal S (2003) Thymidine analogue-sparing highly active antiretroviral therapy (HAART). J HIV Ther 8: 2-6.

28. McComsey GA, Libutti DE, O'Riordan M, Shelton JM, Storer N, et al. (2008) Mitochondrial RNA and DNA alterations in HIV lipoatrophy are linked to antiretroviral therapy and not to HIV infection. Antivir Ther 13: 715-722.

29. Ward WF, Qi W, Van Remmen H, Zackert WE, Roberts LJ, 2nd, et al. (2005) Effects of age and caloric restriction on lipid peroxidation: measurement of oxidative stress by F2-isoprostane levels. J Gerontol A Biol Sci Med Sci 60: 847-851.

30. Hulgan T, Morrow J, D'Aquila RT, Raffanti S, Morgan M, et al. (2003) Oxidan stress is increased during treatment of human immunodeficiency virus infection. Clin Infect Dis 37: 1711-1717.

31. Gupta SK, Shen C, Moe SM, Kamendulis LM, Goldman M, et al. (2012) Worsening Endothelial Function with Efavirenz Compared with Protease Inhibitors: a 12-Month Prospective Study. PloS ONE 7: e45719 